\title{
P I 9-37. Replication-defective lymphocytic choriomeningitis virus vectors boost cellular and humoral immunity after DNA or adenovirus vector priming
}

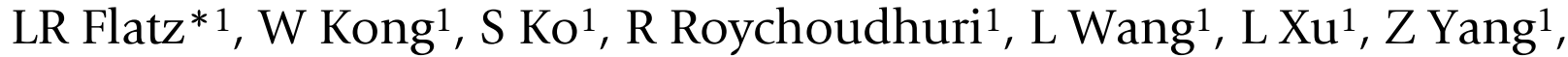 \\ M Honda ${ }^{1}$, DD Pinschewer ${ }^{2}$ and GJ Nabel ${ }^{1}$
}

Address: ${ }^{1}$ Vaccine Research Center/NIAID/National Institutes of Health, Bethesda, MD, USA and ${ }^{2}$ Department of Pathology and Immunology, C.M.U., University of Geneva, Geneva, Switzerland

* Corresponding author

from AIDS Vaccine 2009

Paris, France. 19-22 October 2009

Published: 22 October 2009

Retrovirology 2009, 6(Suppl 3):P357 doi:10.1 I86/1742-4690-6-S3-P357

This abstract is available from: http://www.retrovirology.com/content/6/S3/P357

(c) 2009 Flatz et al; licensee BioMed Central Ltd.

\section{Background}

Immunization against chronic life threatening viral infections such as HIV remains an elusive goal. Data from clinical and non-human primate studies suggest that the generation of potent CD8 $\mathrm{T}$ cell responses as well as broadly neutralizing antibodies will be needed for protective immunity against AIDS. Because of its well known ability to stimulate such immune responses, we have explored the potential of a replication-defective recombinant lymphocytic choriomeningitis virus (rLCMV) vector to elicit antigen-specific immunity to HIV-1.

\section{Methods}

We developed a packaging cell system to produce replication-defective vector and analyzed its ability to replicate in vitro and in vivo. The immunogenicity of this vector was evaluated by comparison of different prime boost vectors encoding HIV-1 envelop (Env) using either DNA, recombinant adenovirus serotype 5 (rAd5) or alternative serotype rAd for priming followed by boosting with a rLCMV vector in mice. T cell immunogenicity was assessed by either analyzing tetramer positive cells or intracellular cytokine staining after peptide stimulation and B cell immunity by ELISA.

\section{Results}

The rLCMV vector was replication-defective, had no pathological consequences in mice injected intracerebrally with vector, and efficiently infected dendritic cells. The immune responses to rLCMV vaccination, alone or after DNA or recombinant rAd5 priming were compared to these vectors alone or to DNA/rAd5 prime boost immunizations. This analysis revealed that rLCMV boosting of DNA or rAd5 elicited Env-specific CD4 and CD8 T-cell ICS responses of greater magnitude and persistence than either vector alone or DNA/rAd5 immunization. Immunogenicity in non-human primate studies is in progress and will be discussed.

\section{Conclusion}

These results suggest that rLCMV represents a vaccine vector that targets dendritic cells and elicits potent and persistent CD8 responses alone or in different prime boost combinations. This vector merits further consideration for clinical development of an AIDS vaccine. 